\title{
A case study of seismograph self-noise test from Trillium 120QA seismometer and Reftek 130 data logger
}

\author{
Weiwei Xu (D) Songyong Yuan
}

Received: 28 July 2019/Accepted: 10 September 2019 /Published online: 6 November 2019

(C) The Author(s) 2019

\begin{abstract}
Seismograph self-noise has become a de facto standard for instrument comparisons and their performance assessment and is considered as one of the most vital parameters for instrument comparison. For self-noise testing of modern force-balance feedback broadband seismometers, several factors have been thoroughly discussed and thought to be attributable to the self-noise estimate, including the data selection criteria, sensor alignment correction, timing error, correlation analysis method, and computational parameter selection during the computational process. This study focuses on some other factors, such as local site conditions, temperature insulating methods, and data logger self-noise interferences, with an aim to differentiate the self-noise contribution of these sources and their dependencies on time and frequency. A series of experiments were conducted at the Beijing National Earth Observatory using a Trillium 120QA seismometer and Reftek130 data acquisition system at three different locations ranging from the ordinary equipment warehouse to global seismographic network level cave with a hardrock base. Results show that noise-free site is necessary for the self-noise test in a frequency band greater than approximately $0.1 \mathrm{~Hz}$. However, for a frequency band
\end{abstract}

W. Xu $(\bowtie) \cdot S$. Yuan

Institute of Geophysics, China Earthquake Administration (CEA), No.5, Minzudaxue Nanlu, Haidian district, Beijing 100081, China e-mail:wwxuww@sina.com

S. Yuan

e-mail: ysy1@vip.sina.com less than $0.1 \mathrm{~Hz}$, the insulation method and installation procedures are far more important, although the influence of the site location cannot be neglected fully. A suitable preamp should be selected in the data logger configurations to ensure that the low-noise amplitude of the sensor signal is above the digitizer noise level.

Keywords Seismograph self-noise · Seismometer testing $\cdot$ Broadband seismometer $\cdot$ Seismic data acquisition system $\cdot$ Seismometer insulation method

\section{Introduction}

Seismograph self-noise defines the lower limit of the seismic noise detection in broadband seismic observations and plays an important role in seismic instrument development and seismic noise analysis. For seismometers with comparatively high self-noise, such as strong-motion accelerometers or lower-grade sensors, it is possible to obtain accurate self-noise estimates through noise power spectra estimation by testing a single senor at a relatively noise-free location when the sensor's self-noise is well above the site noise (Ringer et al. 2015a). For high-quality low-noise broadband sensors, no locations exist where background noise levels are below that of the sensor across a wide frequency range; therefore, the two-sensor method was developed to isolate the self-noise estimates from synchronously recorded data of two collocated sensors, assuming that the internal noises between each channel pair and internal noise and common input signal are uncorrelated (Holcomb 1989; Holcomb 1990). By 
introducing a third collocated sensor, the three-sensor method can estimate sensor self-noise while minimizing errors during the estimation owing to the uncertainty in the transfer functions (Sleeman et al. 2006).

Practically, several factors can influence the test results, and even for the same sensor model, different test results can be obtained if these factors are not considered well (Ringler and Hutt 2010; Yin et al. 2013; Xu et al. 2017). Different coherence analytical techniques, including the two- and three-sensor methods, have been investigated well. The three-sensor method has become the preferred approach for estimating self-noise of broadband sensors owing to its relative stability and robustness in a broad range of frequencies (Ringler et al. 2015b). During the mathematical computation, the Welch method is typically adopted to evaluate the signal power spectral density (PSD), wherein specific parameters, such as sample size, sample rate, bandwidth, and windowing, need to be decided before the computation. Some optimal parameter selections have been put forward for the seismometer self-noise testing by the Guidelines for Seismometer Testing workshops to make the test results comparable in some reasonable manner (Hutt et al. 2009; Evans et al. 2010). Similarly, the effects of these parameters' selection have been well investigated following the statistical examination of 9800 different parameter combinations by some scholars; a zone of reasonable self-noise calculation parameter combinations was identified (Li et al. 2015).

Several studies (Holcomb 1990; Sleeman and Melichar 2012; Tasič and Runovc 2013; Gerner and Bokelmann 2013; Ringler et al. 2015a, 2015b; Gerner et al. 2017) have shown that sensor misalignment is an important source of error during self-noise testing of seismometers based on collocation methods. A synthetic test was conducted to quantify the effect of sensor misalignment as a function of signal-to-noise ratio (SNR) on the self-noise estimate. Results showed that for the higher SNR, the effect of the tiny misalignment might be clarified. This implied that these types of measurements should be performed at seismically quiet locations or the misalignment error must be well corrected before the correlation analysis to avoid the misinterpretation of test result. Considering that an ideal seismically quiet location can hardly be found and misalignment error can hardly be guaranteed to be less than $1^{\circ}$ (Ekström and Busby 2008; Ringler et al. 2013), the correction based on the trace rotation is a good option and different rotational schemes have been developed to correct the effect of sensor misalignment (Tasič and Runovc 2013; Gerner and Bokelmann 2013; Gerner et al. 2017). The timing errors between sensor records contribute to the incoherent-noise estimates and are related to the SNR of the records (Ringer et al., 2011); however, this effect is increasingly negligible because the timing clock inside modern data acquisition systems can be well synchronized once the inside global navigation satellite system (GNSS) module is normally operated.

This study aims to evaluate the performance of seismometers used in the ChinArray project, better understand the operating range of the broadband seismometers, and setup a reasonable testing environment for the instruments. Herein, we mainly focused on the effects of seismometer self-noise testing from local site conditions, temperature insulating methods, and data logger self-noise interference with an aim to differentiate the self-noise contribution of these sources and their dependencies on time and frequency. Then, a reasonable procedure and test environment for a broadband seismometer self-noise test was proposed.

\section{Experimental setup}

In broadband seismometer self-noise testing, the noise estimate of vertical component is typically adopted as the final result since the horizontal output often shows incoherent elevated noise levels because of the local changes in wind or pressure, which are extremely difficult to avoid in most cases. However, for a Galperintype seismometer (Galperin 1955), since the three sensing elements are arranged in a symmetric triaxial manner, each traditional horizontal or vertical output is computed from the combination of three sensing elements using a rotational matrix; therefore, the vertical output can reflect the performance of all three sensing elements. This is one of the reasons we chose Trillium 120QA as our test seismometer. Another reason is that this model is well tested by manufacturer, offering a good reference to justify the test environment and methodology. We used the Reftek 130 data logger as the test digitizer because this model is among the most popular digitizers used in portable seismic observation in China; however, the Q330HR digitizer is mostly recommended in such experiments owing to its high resolution.

At the Beijing National Earth Observatory, we conducted self-noise test experiments comprising Trillium 120Q seismometers and Reftek 130 data loggers at three 
different locations; setups are shown in Fig. 1. Sites 1 and 3 were located in a cave with a hard-rock base, where one of the global seismographic network seismic stations with the code BJT occurs. Site 1 was in a chamber $\sim 50 \mathrm{~m}$ from BJT with the best seismically quiet environment, whereas site 2 was $\sim 100 \mathrm{~m}$ from BJT and much closer to the cave door with median noise surrounding of three. Site 3 was in the equipment warehouse of the China Seismic Array Instrument Center, which was in the middle of a village with the worst seismic background in a normal sense.

The naming of the three experimental sites is simply based on the chronological order, i.e., the tests were conducted first at site 1 . However, the test result was not significantly consistent with the one provided by the manufacturer; hence, experiments at sites 2 and 3 were conducted. In the cave chamber where the site 1 experiment was completed, a granite pier built several decades ago with a $\sim 2 \mathrm{~m}^{2}$ surface and $\sim 70 \mathrm{~cm}$ in height holds a glass tank that is tightly stuck to the pier surface. The three seismometers were placed in this glass tank and a glass plate was then placed as a cover to insulate the sensors from the outside. Some small holes occur at the top of the tank wall for cables to travel between the seismometers and data loggers and they were well sealed using butter following the placement. The upper right picture in Fig. 1 shows the case before the glass cover was placed. At site 2, three seismometers were placed on a rigid granite slab $1000 \times 1000 \times 15 \mathrm{~cm}$ in size, which was supported at three points with lead pads on the floor of the instrument warehouse. As distinguished from site 1, each seismometer was individually insulated using a special cover developed by Nanometrics Inc. Afterwards, an overall cover comprising polyethylene foam was placed over all the sensors and pier; additionally, a thick blanket was placed over the equipment and down the sides of the concrete pier. At site 3 , the pier was nearly the same as that of site 1 except for a slightly smaller surface size and insulation method was nearly the same as that of site 2 . The lower left picture in Fig. 1 shows the case before the overall cover was placed at site 2 and lower right one shows the case after all the insulation procedures were completed at site 3 .
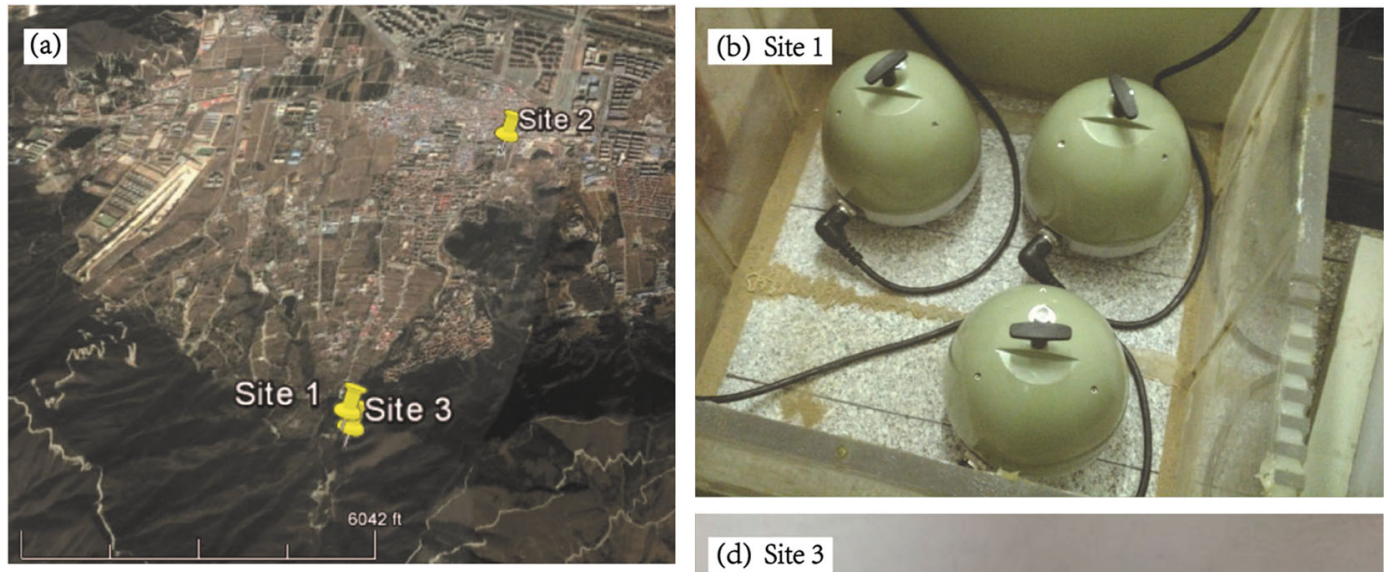

(d) Site 3
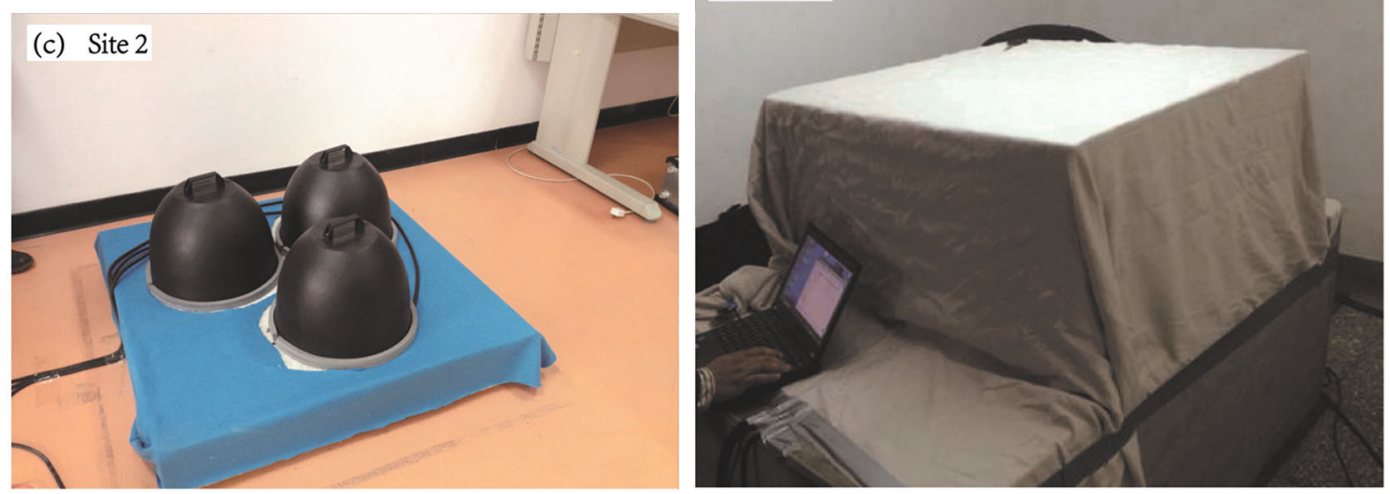

Fig. 1 aGoogle map of the Beijing National Earth Observatory showing the locations of the three experiments for seismometer self-noise testing. (b), (c), and (d) show the seismometer deployment view of each experiment herein 
To isolate the seismometer self-noise, it is critical that the digitizer has a self-noise level well below that of the seismometer. Most current data loggers in the market typically offer several options of preamps for lowernoise amplification of the sensor and user can select a suitable preamp based on their own observational purpose. Taking the Reftek 130 as an example, each channel can be configured as unity or high gain, i.e., low or high preamp electronics selected to have different selfnoise levels, respectively. To clarify the interferences of digitizer self-noise with the seismometer, we intentionally configured this option differently during our experiments at the three sites, i.e., we selected high gain at site 1 and unity gain at sites 2 and 3 . All the digitizers were configured to be continuously synchronized from a GNSS signal and record two streams at a sample rate of 200 samples and 1 sample per second (sps), respectively, as shown in Table 1. Altogether, seven data loggers were used for the same three seismometers mainly because the three data loggers used at site 1 had been delivered to the field for use following the test, whereas one of the three data loggers at site 2 was substituted by another at site 3 because of maintenance.

\section{Analysis and results}

We employed the three-sensor method to isolate the seismometer self-noise based on the continuous record of three seismometers; the methodology details can be found in a previously published paper (Sleeman et al. 2006). Although data selection had been considered as an important item to estimate self-noise, no criteria or agreement exists. Some random popcorn-like noises owing to cable stress release or semiconductor defects, which definitely belong to instrument self-noise, are difficult to describe. Most researchers claim that only quiet time periods, such as nighttime without special seismic or other events, should be used for analysis. Some scholars have attempted to combine continuous and special data selection criteria such as defining the threshold between self-noise statistics and its mode (Sleeman and Melichar 2012). Herein, instead of only analyzing the noise-free data, we obtained self-noise estimation variations during the entire test periods. Herein, we wished to see how the local environmental conditions affected the self-noise test results using different insulation methods and how these effects varied with time and frequency.

We corrected the potential misalignment angles between seismometers based on three-dimensional (3D) rotational algorithm of raw seismic traces to maximize coherence, which is similar in principle to that of previous studies (Tasič and Runovc 2013; Gerner et al. 2017). The raw data were deconvolved with instrument response of both seismometers and data loggers. For the spectrum estimation, we adopted the parameters recommended in a previous study (Evans et al. 2010) for the Welch estimation (Welch 1967), which includes $2^{19}$ and $2^{15}$ sampling point duration for 200 and $1 \mathrm{~Hz}$ stream, respectively, and a constant overlap of $87.5 \%$ of the window length chosen. Upon combining the test results of two different sample rates, the self-noise estimates were obtained as a function of frequency from 0.0005 to $50 \mathrm{~Hz}$. Finally, a 25\% logarithmic smoothing scheme

Table 1 Different self-noise test configurations used at three sites. All tests were run for at least one week to ensure sensors had settled. The serial number of data loggers and sensors are indicated in the parenthesis of each model used

\begin{tabular}{llll}
\hline Site & Data loggers & Sensors & Description \\
\hline Site 1 & $\begin{array}{l}\text { Reftek 130, High gain } \\
\text { (B00E, B008, AF83) }\end{array}$ & $\begin{array}{l}\text { Trillium 120QA } \\
(1880,1881,1882)\end{array}$ & $\begin{array}{c}\text { At a cave chamber with the least cultural and environmental noise } \\
\text { interferences of three sites. } \\
\text { All seismometers in a well-sealed glass tank as insulation cover on } \\
\text { an } 80 \text { cm high granite pier }\end{array}$ \\
Site 2 & $\begin{array}{l}\text { Reftek 130, Unity gain } \\
\text { (9B13, AF80, B044) }\end{array}$ & $\begin{array}{l}\text { Trillium 120QA } \\
(1880,1881,1882)\end{array}$ & $\begin{array}{c}\text { At the equipment warehouse of China Seismic Array Instrument } \\
\text { Center with the largest noise interferences of three sites. } \\
\text { Each seismometer individually insulated with a cover designed by } \\
\text { Nanometrics Inc. and polyethylene foam used as the whole } \\
\text { thermal insulation for all three seismometers on a 15 cm high } \\
\text { granite pier }\end{array}$ \\
& $\begin{array}{l}\text { Reftek 130, Unity gain } \\
\text { (B031, AF80, B044) }\end{array}$ & $\begin{array}{l}\text { Trillium 120QA } \\
(1880,1881,1882)\end{array}$ & $\begin{array}{c}\text { At a cave chamber with the intermediate noise interferences } \\
\text { of three sites. } \\
\text { Thermal insulations similar with site 2. }\end{array}$ \\
\end{tabular}


was applied to smoothen the final results (Ringler and Hutt 2010).

PSD presentation of the seismometer self-noise tested at the three sites is shown in Fig. 2, where only the vertical test results are presented. For all the test results shown in Fig. 2, we found limited abnormal elevation of self-noise curves in the microseism frequency band, or in other words, limited leakage of microseism noise into the self-noise estimates (Gerner et al. 2017); this result proved that our misalignment procedures worked well. Results showed that sites 1 and 3 in the cave chambers have more stable results with a smaller distributional range than those in site 2 . Theoretically, the minimum self-noise curve in the figure represents the best test result during the entire test period and apparent differences can be seen for three sites in the entire frequency band. Some abnormal scattering points were found even after the smoothing average at less than $0.002 \mathrm{~Hz}$, which might have been caused by the relatively fewer points involved in the averaging and possible numerical instabilities in computation. In each result shown in the figure, a curve termed as the 10th\% self-noise is shown; it was defined as the 10th percentile of the self-noise statistics of all results in each test, with a similar meaning as the commonly used power probability density (McNamara and Buland 2004). Figure 3 showed the temporal variations in self-noise statistics at the three sites. Again, site 1 showed the most

(a) Site 1

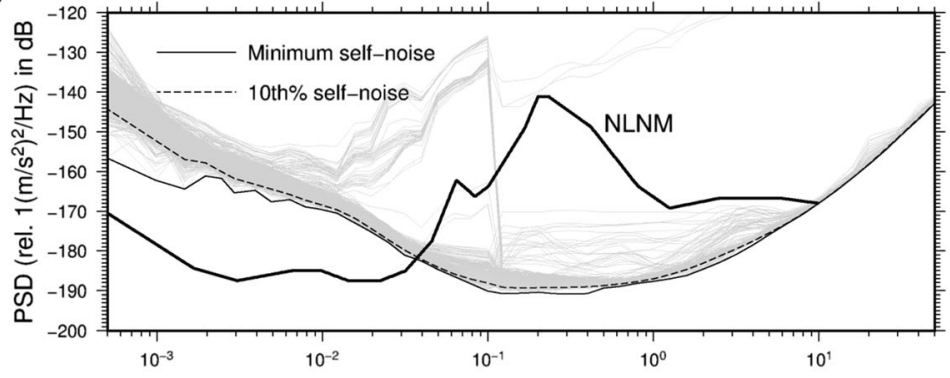

(b)

Site 2

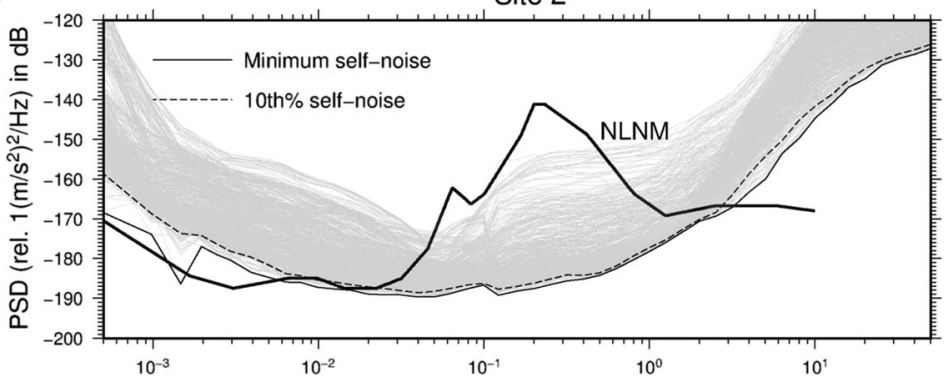

(c)

Site 3

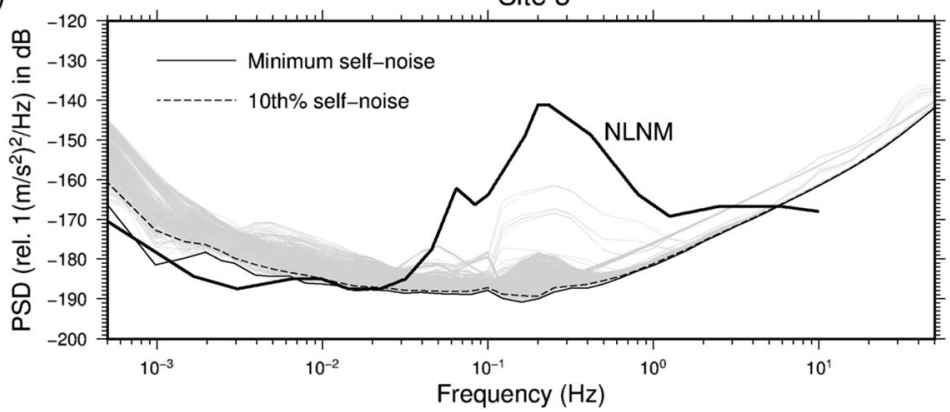

Fig. 2 PSD presentation of seismometer self-noise for an approximately 1-week long seismic data experiment at sites 1 (a), 2 (b), and 3 (c) computed using vertical records. The thin gray line is the self-noise PSD computed from each data segment; thick solid black line is the new low-noise model (NLNM) of Peterson (1993); thin solid black line is the minimum self-noise curve from 1-week-long evaluation results; and dashed thin line is the 10th percentile of all self-noise curves 
stable result except for a seismic event contamination between day 203 and 204 and several other smaller events. Site 2 results showed strong diurnal changes that are closely related to nearby human activities. Site 3 results were more stable than those of site 2; however, they were less stable than those of site 1 , which is consistent with their overall intuitive seismic background noise conditions.

To quantitatively compare the test results of these three sites, we plotted all the results in the same figure, as shown in Fig. 4. The minimum and 10th\% self-noise of each site are shown with different color and line types together with the result provided by the manufacturer. Additionally, we manually selected a seismically quiet segment during the local midnight period for each test and computed the self-noise at these specific times, which are termed as sample self-noises in the figure.
We can see that for the frequency band less than $0.1 \mathrm{~Hz}$, both the 10 th $\%$ and sample self-noise for sites 2 and 3 were consistent with the manufacturer's result, whereas the results for site 1 were $0-15 \mathrm{~dB}$ higher. However, for the frequency band greater than $0.1 \mathrm{~Hz}$, only the results of site 1 were consistent with the manufacturer's result and the other two sites showed strong differences. We adopted different preamp options in the Reftek 130 data loggers; therefore, we also measured these data loggers' self-noises at different configurations and plotted the test results in the figure. Seven data loggers were tested over one night by terminating the input connecters with $50-\Omega$ resistors to simulate the output impedance of the Trillium 120QA seismometer. As shown in the figure, the self-noises using the high gain preamp option in Reftek 130 showed a significantly lower level than the seismometers, whereas the unity gain option result
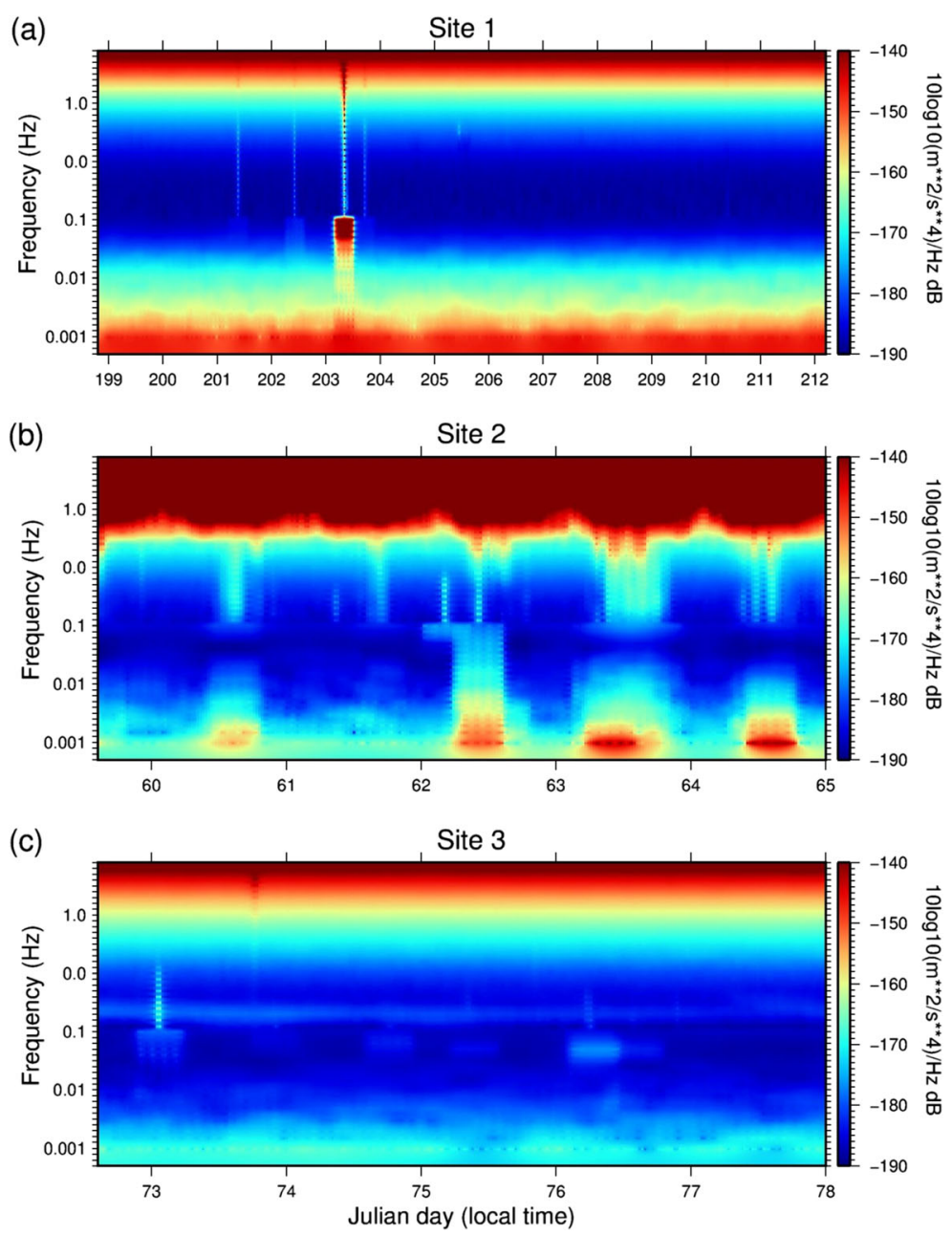

Fig. 3 Temporal variation in seismometer self-noise PSDs for (a) sites 1, (b) 2, and (c) 3 


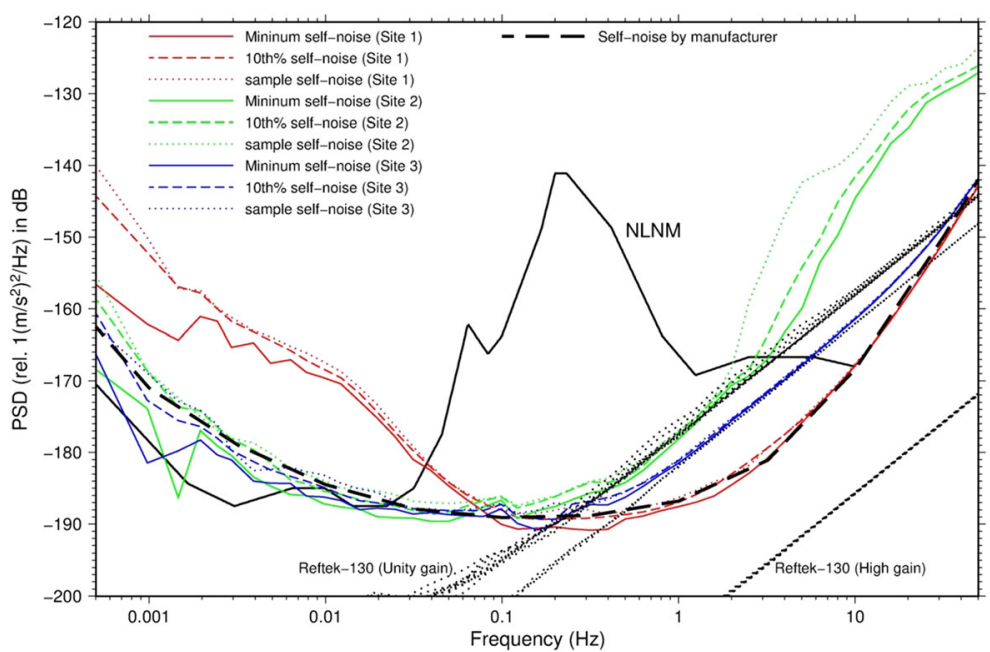

Fig. 4 Measured self-noise comparison between seismometers from three different sites, data loggers with different preamp options, and manufacturer's nominal curve. The minimum selfnoise represents the minimum value of all three seismometers during the entire test period at a site; $10 \%$ minimum self-noise is the 10th percentile of all three seismometers' test results; and the sample self-noise curve is the analytical result from a visually selected quiet data segment crisscrossed with the seismometer's result at some frequency bands, suggesting that high gain option is necessary in the self-noise testing of low-noise broadband seismometers. Another interesting result is that we found a $\sim 4 \mathrm{~dB}$ self-noise difference in different individual data loggers for the same unity gain preamp option, which might reflect some minor modifications in the electronics of the data loggers.

\section{Discussion and conclusions}

Several influential factors affect the final test results of a low-noise broadband seismometer self-noise test, including the data selection criteria, sensor alignment correction, correlation analysis method, and computation parameter selection during the computation process. A seismically "quiet" site is necessary for the frequency band greater than approximately $0.1 \mathrm{~Hz}$; however, for the frequency band less than $0.1 \mathrm{~Hz}$, the thermal insulation method and installation procedures are more important; however, the influence of site location cannot be fully neglected. When a Reftek 130 model was used as the data logger in a seismometer self-noise test, the high gain preamp option should be adopted to ensure that the self-noise of the sensor is at a level above the digitizer noise level, thereby not interfering with the sensor's test result. As a part of the entire observational system, data loggers should select a suitable preamp option to match the seismometers used based on different observational purposes.

Once all the aforementioned factors were fully considered, the self-noise test results of Trillium 120QA were found to be consistent with the manufacturer's nominal curve at high- and low-frequency bands, respectively. We concluded that the elevated self-noise in the low-frequency noise at site 1 might have originated from two sources: 1 ) poor insulation setup that can be improved by either additional individual covers for each sensor at sites 2 and 3 or other effective insulation measures such as the whole neoprene insulation method (Sleeman and Melichar 2012) and 2) the nonnegligible contribution of the heavy insulation glass box to the underlying rock base, which made the sensor measurements uneven between each other. Test results showed that the frequency below $0.1 \mathrm{~Hz}$ at site 1 could be improved to a nominal value after similar thermal insulation and installation procedures were applied at sites 2 and 3, i.e., removing the current heavy glass box, leaving only the bare clean surface of the concrete pier, directly placing the sensors on the concrete pier with individual covers over them, placing an overall insulating cover to fit over this and sensors, and placing a thick blanket over everything, including the downsides of the concrete pier. However, these procedures will be implemented during our future experiment when completing such seismometer self-noise tests. 
Theoretically, low site noise is not the necessary condition for a seismometer self-noise test based on the correlation analysis method because this method only requires the ground motion input of each sensor to be as consistent as possible among each other. Results showed that the low- and high-frequency self-noise of the seismometer had different sensitivities to the surrounding test conditions. For example, the lowfrequency self-noise tested at site 2 with a better insulation procedure showed a lower level than that at site 1 , even though the site noise level at site 1 was far more ideal than that at site 2. However, we do not suggest that good site selection is not important in a seismometer self-noise test; the good high-frequency self-noise result obtained at site 1 showed the importance of a good background noise environment in this frequency band. Additionally, as shown in Fig. 4, there was an improved level at site 3 than that at site 2 for the low-frequency self-noise, even though they shared nearly the same insulation setup. We must emphasize on the sensor insulation method in seismometer self-noise testing, particularly for the low-frequency band. Herein, we compared the PSD results at three sites, as shown in Fig. 5, to illustrate their absolute noise levels. It is clear that the PSD of site 1 also showed an obvious elevation in the low-frequency band noise than that of the other two sites, which both had some low-noise levels mostly during the local nighttime. This partially explained the final elevated self-noise results at site 1 .
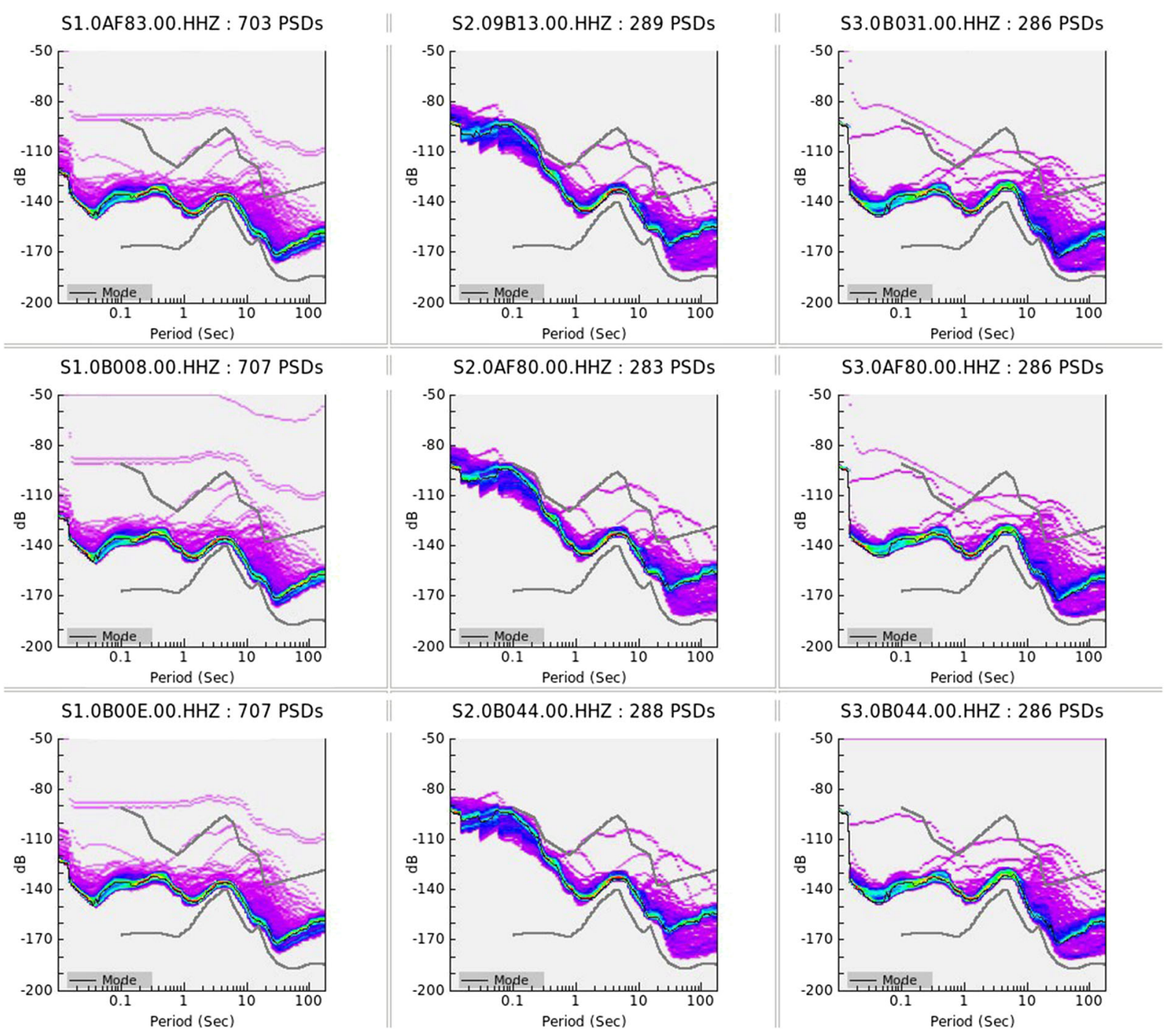

Fig. 5 Noise PDFs obtained for seismometer vertical components at sites 1 (“S1”), 2 (“S2"), and 3 (“S3”) using the PQLX package 
Acknowledgements The authors would like to thank Nanometrics Inc. for useful discussions and help with the loan of their specially designed covers and an anonymous reviewer for the suggestions and comments that helped improve the manuscript. This work was mainly supported by the National Key R\&D Program of China (Grant Number 2017YFC1500201) and was partially supported by the National Natural Science Foundation of China (Grant Number 41474047).

Open Access This article is distributed under the terms of the Creative Commons Attribution 4.0 International License (http:// creativecommons.org/licenses/by/4.0/), which permits unrestricted use, distribution, and reproduction in any medium, provided you give appropriate credit to the original author(s) and the source, provide a link to the Creative Commons license, and indicate if changes were made.

\section{References}

Ekström G, Busby RW (2008) Measurements of seismometer orientation at USArray transportable Array and backbone stations. Seismol Res Lett 79(4):554-561

Evans JR, Followill F, Hutt CR, Kromer RP, Nigbor RL, Ringler AT, Steim JM, Wielandt E (2010) Method for calculating selfnoise spectra and operating ranges for seismographic inertial sensors and recorders. Seismol Res Lett 81(4):640-646

Galperin EI (1955) Azimuthal method of seismic observations (in Russian). Gostoptechizdat 80

Gerner A, Bokelmann G (2013) Instrument self-noise and sensor misalignment. Adv Geosci 36:17-20

Gerner A, Sleeman WL, Grasemann B (2017) Improving selfnoise estimates of broadband seismometers by $3 \mathrm{D}$ trace rotation. Seismol Res Lett 88(1):1-8

Holcomb LG (1989) A direct method for calculating instrument noise levels in side-by-side seismometer evaluations, U.S. Geol. Surv. Open-file Rept. No. U.S. Geological Survey, Albuquerque, New Mexico, pp 89-214

Holcomb LG (1990) A numerical study of some potential sources of error in side-by-side seismometer evaluations, U.S. Geol. Surv. Open-file Rept. No. U.S. Geological Survey, Albuquerque, New Mexico, pp 90-406

Hutt CR, Evans JR, Followill F, Nigbor RL, Wielandt E (2009) Guidelines for standardized testing of broadband seismometers and accelerometers. US Geol Surv Open-File Rept 20091295:62

Li X, Yang D, Xie J, Ma J, Yuan S, Xu W, Zhao J, Li D (2015) Applicability of the Welch method for examining self-noise level parameters for broadband seismometers. J Geodes Geodyn 6(3):233-239
McNamara DE, Buland RP (2004) Ambient noise levels in the continental United States. Bull Seismol Soc Am 94(4):15171527

Ringler AT, Hutt CR (2010) Self-noise models of seismic instruments. Seismol Res Lett 81(6):972-983

Ringler AT, Hutt CR, Evans JR, Sandoval LD (2011) A comparison of seismic instrument self-noise analysis techniques. Bull Seismol Soc Am 101:558-567. https://doi.org/10.1785 $/ 0120100182$

Ringler AT, Hutt CR, Persefield K (2013) Seismic station installation orientation errors at ANSS and IRIS/USGS stations. Seismol Res Lett 84(6):926-931

Ringler AT, Evans JR, Hutt CR (2015a) Self-noise models of five commercial strong-motion accelerometers. Seismol Res Lett 86(4):1143-1147

Ringler AT, Sleeman R, Hutt CR, Gee LS (2015b) Seismometer self-noise and measuring methods. In: Beer M, Kougioumtzoglou IA, Patelli E, Au S-K (eds) Encyclopedia of earthquake engineering. Springer, Berlin/Heidelberg, Germany, pp 3220-3231

Peterson J (1993) Observations and modeling of seismic background noise. USGS Open-File Report 93-322, 94pps

Sleeman R, Melichar P (2012) A PDF representation of the STS-2 self-noise obtained from one year of data recorded in the Conrad Observatory, Austria. Bull Seismol Soc Am 102: $587-597$

Sleeman R, van Wettum A, Trampert J (2006) Three-channel correlation analysis: a new technique to measure instrumental noise of digitizers and seismic sensors. Bull Seismol Soc Am 84(1):222-228

Tasič I, Runovc F (2013) Determination of a seismometer's generator constant, azimuth, and orthogonality in threedimensional space using a reference seismometer. J Seismol 17(2):807-817

Welch PD (1967) The use of fast Fourier transform for the estimation of power spectra: a method based on time averaging over short, modified periodograms, IEEE Trans Audio Electroacoust. AU-15, 2, 70-73

Xu W, Yuan S, Ai Y, Xu T (2017) Applying multiple channel correlation technique in the self-noise measurement of broadband seismographs. Chinese J Geopphys (in Chinese 60(9): 3466-3474. https://doi.org/10.6038/cjg20170916

Yin X, Chen J, Li S, Guo B (2013) The research on self-noise measurement method of moveable broadband seismometer. Seismology and Geology (in Chinese) 35(3):576-583

Publisher's note Springer Nature remains neutral with regard to jurisdictional claims in published maps and institutional affiliations. 